DOI: $10.5216 /$ cab.v14i4.16377

\title{
INFLUÊNCIA DE PROCEDIMENTOS GINECOLÓGICOS FREQUENTES NO DESEMPENHO PRODUTIVO E REPRODUTIVO DE FÊMEAS BUBALINAS MURRAH
}

\author{
MARILIA VIVIANE SNEL-OliveIRA ${ }^{1}$, DANIEla WETZEL-GASTAL ${ }^{2}$, ROBERTO SARTORI ${ }^{3}$, JAIRo PEREIRA \\ NEVES $^{4}$

\begin{abstract}
1Professora Doutora da Faculdade União Pioneira de Integraçao Social, Brasília, DF, Brasil. mariliavso@gmail.com

2Professora da Faculdade de Medicina Veterinária - Universidade Técnica de Lisboa, Lisboa, Portugal. 3Professor Doutor da Escola Superior de Agricultura "Luiz de Queiroz" - ESALQ, Universidade de São Paulo, Piracicaba, SP, Brasil

4Professor Doutor da Faculdade de Medicina Veterinária - Universidade de Brasília, Brasilia, DF, Brasil.
\end{abstract}

\section{RESUMO}

Este trabalho teve o objetivo de avaliar, em búfalas Murrah, a interferência da manipulação diária dos animais para exame ginecológico em parâmetros reprodutivos e produtivos. Vinte e quatro búfalas, paridas no outono, ordenhadas uma vez ao dia, mantidas a pasto foram distribuídas nos grupos: Pesquisa (GP; n=13); e Controle (GC; $n=11)$. As fêmeas do GP, após a ordenha, foram diariamente, desde o $7^{\circ}$ dia pós-parto até o primeiro estro e cobertura, conduzidas até o curral e submetidas a exame ginecológico e coleta de sangue. Os animais do GC, após a ordenha, foram liberados diretamente para o pasto sem nenhuma manipulação. O GP apresentou intervalo partoprimeiro estro menor $(40,4 \pm 9,0$ dias $)$ que o $\mathrm{GC}(59,2 \pm 24,4$ dias; $\mathrm{P}<0,05)$. Não foi observada diferença no intervalo parto-concepção e na taxa de prenhez ao final da estação reprodutiva $(61,2 \pm 21,3$ dias e $86,0 \%$ GP; $71,2 \pm 30,4$ dias e $91,0 \% \mathrm{GC}$, respectivamente; $\mathrm{P}>0,05)$, bem como na produção de leite. O número de coberturas por concepção foi maior no GP $(2,1 \pm 0,9)$ do que no GC $(1,5 \pm 0,5$; $\mathrm{P}<0,05)$. Conclui-se que a manipulação diária dos animais para pesquisa empregada neste experimento afeta alguns parâmetros reprodutivos, comprometendo o número de coberturas por concepção, mas não interfere no intervalo parto-concepção, na taxa de prenhez ao final da estação e na produção leiteira, considerando-se o sistema de manejo e os índices de produção do experimento.

PALAVRAS-CHAVE: búfalo; exame ginecológico; leite; pesquisa; reprodução.

\section{INFLUENCE OF FREQUENT GYNECOLOGICAL PROCEDURES ON REPRODUCTIVE AND PRODUCTIVE PERFORMANCE OF MURRAH BUFFALO FEMALES}

\section{ABSTRACT}

This study aimed to evaluate the interference of daily gynecological exam on reproductive and productive parameters in Murrah buffaloes. Twenty-four buffaloes, which have calved in the autumn season, were milked once a day and kept on pasture, were divided into two groups: Research (RG; n=13) and Control (CG; n=11). The animals in RG were daily taken to the corral and submitted to gynecological examination and blood collection immediately after milking, from the $7^{\text {th }}$ day postpartum until the first estrus and breeding day. Animals in CG were released directly to pasture after milking without any manipulation. The RG had smaller calvingfirst estrus interval (40.4 \pm 9.0 days) than CG (59.2 \pm 24.4 days; $\mathrm{P}<0.05)$. There was no difference in calvingconception interval and pregnancy rate at the end of the reproductive season $(61.2 \pm 21.3$ days and $86.0 \%$ RG; 
$71.2 \pm 30.4$ days and $91.0 \% \mathrm{CG}$, respectively; $\mathrm{P}>0.05$ ), as well as in milk production. The number of mating per conception was higher in RG $(2.1 \pm 0.9)$ than in CG $(1.5 \pm 0.5 ; \quad \mathrm{P}<0.05)$. We concluded that the daily management of animals used for research in this experiment affects some reproductive parameters, such as the number of mating per conception, but it does not affect calving-conception interval nor the pregnancy rate at the end of the season, as well as milk production, considering the management system and the production indices of the experiment.

KEYWORDS: buffalo; gynecological exam; milk; research; reproduction.

\section{INTRODUÇÃO}

Os búfalos de rio (Bubalus bubalis fluviatilis ou bubalis; $2 \mathrm{n}=50$ cromossomos) são uma fonte viável de proteína de origem animal cada vez mais procurada pelo consumidor. O búfalo (Bubalus bubalis) tem a capacidade única de adaptar sua necessidade de energia, reduzindo-a quando a disponibilidade na dieta é limitada, mantendo seu peso vivo e sua função reprodutiva (CAMPANILE et al., 2010). Esses animais têm sido criados com o propósito principal de produção de leite como também, para produção de carne e trabalho e estão presentes em todos os continentes (PAUL \& LAL, 2010).

Essa espécie tem despertado a atenção de criadores, consumidores e pesquisadores, não só nas regiões onde é tradicionalmente criada, mas também em outras áreas, aumentando o interesse pela criação e pela melhoria no desempenho produtivo e reprodutivo. Alguns relatos no Brasil (VALE \& RIBEIRO, 2005; BERNARDES, 2007) demonstram a adaptação dessa espécie às condições ambientais brasileiras, bem como a necessidade de seleção e manejo adequado, como qualquer espécie animal.

O conhecimento da fisiologia reprodutiva da espécie é de fundamental importância tanto para a obtenção de índices reprodutivos elevados e uma boa rentabilidade na produção quanto para o emprego das biotecnologias da reprodução. Em muitos casos, para a realização de pesquisas no âmbito reprodutivo, faz-se necessário manipular os animais com frequência diária, o que pode afetar os índices reprodutivos e/ou produtivos.

No Brasil, muitas vezes as pesquisas são realizadas em propriedades comerciais, em parceria com os produtores rurais, por escassez de rebanhos experimentais. Entretanto, os rebanhos experimentais existem para atender às pesquisas, já os comerciais visam ao lucro e, para tanto, é necessário o máximo desempenho. Os índices produtivos dos animais envolvidos em pesquisa são passíveis de serem influenciados pelo estresse provocado pela manipulação dos animais. Como manipulação entende-se, por exemplo, deslocamento e manutenção no curral e tronco de contenção, coleta de sangue, pesagem, exame ginecológico, aplicação de fármacos ou placebo (grupos experimentais e controle respectivamente).

Como em experimentos tanto o grupo controle como o grupo experimental devem sofrer o mesmo tipo de manipulação para que se possa avaliar o efeito exclusivo do que está sendo testado, não é possível saber o quanto a manipulação por si só afeta o desempenho animal. Assim, atividades de pesquisa em parceria com criadores podem ser limitadas por não se ter informações mais acuradas para se fornecer ao potencial produtor parceiro sobre o impacto causado pelo experimento no desempenho reprodutivo e produtivo dos animais envolvidos e consequente possível perda econômica. Há, então, a necessidade de se saber o quanto a manipulação por si só pode afetar o desempenho animal.

O objetivo deste trabalho foi avaliar em fêmeas bubalinas da raça Murrah a interferência da manipulação do exame ginecológico diário e da venopunção em parâmetros reprodutivos e produtivos.

\section{MATERIAL E MÉTODOS}

Foram utilizadas 24 búfalas Murrah que tiveram partos eutócicos durante o outono, com escores de condição corporal entre 3,5 e 4,0 (1 = muito magra, $5=$ muito gorda) e que foram separadas dos bezerros $24 \mathrm{~h}$ após o parto. As búfalas foram mantidas em um mesmo pasto e suplementadas com sal mineral antes e após o parto, durante toda a lactação. $\mathrm{O}$ experimento foi realizado na Fazenda Experimental Lagoa Bonita da UPIS Faculdades Integradas, em Brasília, DF, local onde as búfalas nasceram e foram criadas.

As búfalas foram distribuídas em dois grupos: Grupo Pesquisa (GP; $n=13$ ) submetido a 
manipulações; e Grupo Controle (GC; n=11) liberado para o pasto após a ordenha sem sofrer nenhum tipo de manipulação. As búfalas do GP foram conduzidas diariamente, após a ordenha, desde o $7^{\circ}$ dia pós-parto até a manifestação do primeiro estro e acasalamento, até o curral de manejo, onde permaneceram em média por 3 horas e foram submetidas a exame ginecológico (palpação retal para avaliação de útero e ovários e ultrassonografia transretal para avaliação dos ovários) no tronco de contenção. Para diminuir o estresse, foram oferecidas $200 \mathrm{~g}$ de concentrado comercial com $20 \%$ de proteína em um cocho no chão, à frente do animal, durante a palpação retal e exame ultrassonográfico. A cada 24 ou 48 horas, assim que o animal era contido, eram coletadas amostras de sangue por venopunção da jugular, com o objetivo de provocar o estresse da coleta sanguínea, ato realizado em grande quantidade de experimentos. As búfalas do GC foram liberadas para o pasto após a ordenha sem sofrer nenhum tipo de manipulação.

Os animais foram ordenhados uma vez ao dia, com ordenhadeira mecânica (Sistema Espinha de Peixe, GEA Westfalia Surge do Brasil, Gea Farm Technologies do Brasil, Indústria e Comércio de Equipamentos Agrícolas e Pecuários Ltda, Jaguariuna,SP), sem bezerro ao pé, junto com os demais animais do rebanho. O leite produzido foi mensurado uma vez por semana (Mark 5 Milk Meter, Waikato Milking Systems NZ Ltd, Hamilton, New Zealand).

A detecção do estro foi realizada com auxílio de rufião com desvio lateral do pênis utilizando buçal marcador, mantido durante todo o período experimental com as búfalas. As observações foram realizadas duas vezes por dia.

A partir da manifestação do primeiro estro, as fêmeas foram acasaladas por um mesmo touro, de fertilidade comprovada, por até 3 estros, utilizando-se o sistema de monta natural controlada. $\mathrm{O}$ diagnóstico de gestação foi realizado por palpação retal 40-50 dias após a monta.

Foi realizada análise de variância para analisar as diferenças entre os grupos para o intervalo entre o parto e o primeiro estro pósparto, o intervalo entre o parto e a concepção, o número de coberturas por concepção e a produção média de leite diária e total da lactação. A taxa de concepção ao final da estação foi comparada pelo Teste do Qui-quadrado. Todas as análises estatísticas foram realizadas usando-se o software
STATISTICA versão 7 (STATSOFT, INC., 2004).

\section{RESULTADOS E DISCUSSÃO}

Este experimento relata, pela primeira vez, o efeito dos procedimentos ginecológicos realizados diariamente para pesquisa sobre o desempenho produtivo e reprodutivo das búfalas.

$\mathrm{O}$ primeiro estro após o parto foi mais precoce $(\mathrm{P}<0,05)$ nos animais do GP do que no GC (Tabela 1); no entanto, o intervalo entre o parto e o primeiro estro dos dois grupos está dentro das médias relatadas em outros estudos com búfalas de rio (USMANI et al., 1990; BARUSELLI et al., 1996; ABDALLA, 2003). HONPARKHE et al. (2008) testaram a massagem ovariana em dias alternados, durante 25 dias, para estimular a atividade ovariana em búfalas por mais de 60 dias em anestro e não obtiveram sucesso. No presente trabalho, a manipulação do trato reprodutivo foi iniciada bem mais cedo, quando ainda não havia se estabelecido um quadro de anestro, e não se restringiu aos ovários, pois a manipulação não tinha como objetivo massagear os ovários e sim examinar útero e ovários. O efeito benéfico de uma massagem uterina e ovariana por 2 a 3 minutos em vacas que estão em anestro por mais de 60 dias já foi relatado; entretanto, o mecanismo pelo qual a massagem estimula o reinício da atividade ovariana não está claramente entendido (MWAANGA et al., 2004).

$\mathrm{O}$ intervalo parto - concepção do GP e do GC (Tabela 1) foi semelhante $(\mathrm{P}>0,05)$, embora um número maior de coberturas $(\mathrm{P}<0,05)$ tenha sido necessário no GP para que isso acontecesse. É possível que a menor fertilidade do primeiro estro do GP seja devido à sua precocidade, não tendo acontecido ainda a total recuperação do sistema reprodutor e/ou devido à manipulação do aparelho reprodutor inclusive no dia da cobertura. Esse é um importante fator a ser considerado quando se realiza esse tipo de trabalho de pesquisa, devendose, nesses casos, evitar a cobertura ou utilizar sêmen de menor valor comercial quando $o$ intervalo parto - primeiro estro for em torno de 40 dias.

A taxa de prenhez ao final da estação (Tabela 1) foi a mesma para o GP e o GC (P > $0,05)$. Essa taxa de prenhez acima de $85 \%$, aliada ao intervalo parto - concepção de 60 a $70 \mathrm{~d}$, permitindo um intervalo entre partos de 12 a 13 meses, demonstra a capacidade produtiva dessa espécie e sua adaptação às condições ambientais 
do centro-oeste.

$\mathrm{O}$ estresse provocado pela manipulação e contenção dos animais, assim como a diminuição do tempo de pastejo diário, devido à permanência no curral por aproximadamente 3 horas diárias, por um período médio de 40 dias, não interferiram na produção de leite (Tabela 1). Isso indica que os animais tiveram tempo de pastejo suficiente para a ingestão de alimentos, o que é justificável por ter sido utilizada uma única ordenha diária e a produção de leite não ser alta (Tabela 1).

No Brasil existem relatos de produção individual de até $5.142 \mathrm{~kg}$ de leite por lactação (BERNARDES, 2007), de médias de rebanho de $2.130,8 \pm 535,6 \mathrm{~kg} /$ lactação (SAMPAIO NETO et al., 2001) e de média ajustada para 270 dias de $1.635,1 \mathrm{~kg} /$ lactação (SILVA et al., 2010). Comparando-se com as produções citadas acima, a deste rebanho é baixa, mas próxima à produção relatada em vários trabalhos nacionais (JORGE et al., 2002; ANDRIGHETTO et al., 2005; RASSI et al, 2009). É importante ressaltar que a maior parte da lactação dos animais do presente trabalho ocorreu no período de seca, sem suplementação alimentar e com uma única ordenha ao dia.

Não são apresentados resultados de análises sanguíneas, pois as coletas foram realizadas somente no GP com o objetivo de provocar o estresse de uma atividade frequente em experimentação envolvendo reprodução, quando há interesse na caracterização da flutuação endócrina dos animais.

Apesar de o concentrado (200 g) ofertado com o objetivo de manter o animal ocupado durante o exame ginecológico e, assim, diminuir o estresse ter sido fornecido diariamente, o consumo não foi regular, houve uma grande variação entre os animais e entre dias. Desse modo, não foi possível estabelecer nenhum padrão de comportamento e consequente influência sobre os parâmetros avaliados.

Tabela 1 - Média (desvio padrão) ou frequência das variáveis reprodutivas e produtivas avaliadas em búfalas submetidas ou não à manipulação para pesquisa

\begin{tabular}{|c|c|c|c|}
\hline Variáveis & $\begin{array}{l}\text { Grupo Pesquisa } \\
\quad \mathrm{N}=13\end{array}$ & $\begin{array}{l}\text { Grupo Controle } \\
\qquad \mathrm{N}=11\end{array}$ & P-valor \\
\hline Intervalo parto- $1^{\circ}$ estro (dias)* & $\begin{array}{l}40,4^{\mathrm{a}} \\
(9,0)\end{array}$ & $\begin{array}{l}59,2^{\mathrm{b}} \\
(24,4)\end{array}$ & 0,02 \\
\hline Intervalo parto-concepção (dias)* & $\begin{array}{c}61,2 \\
(21,3)\end{array}$ & $\begin{array}{c}71,2 \\
(30,4)\end{array}$ & ns \\
\hline $\begin{array}{l}\text { Taxa prenhez ao final da estação reprodutiva } \\
(\%)^{* *}\end{array}$ & 86,0 & 91,0 & ns \\
\hline $\mathrm{N}^{\circ}$ coberturas/concepção* & $\begin{array}{l}2,1^{\mathrm{a}} \\
(0,9)\end{array}$ & $\begin{array}{l}1,5^{\mathrm{b}} \\
(0,5)\end{array}$ & 0,04 \\
\hline Produção média de leite $(\mathrm{kg} / \mathrm{dia})^{*}$ & $\begin{array}{l}4,0 \\
(0,7)\end{array}$ & $\begin{array}{c}4,3 \\
(0,6)\end{array}$ & ns \\
\hline Produção total de leite (kg/animal/lactação)* & $\begin{array}{l}1263,4 \\
(290,0)\end{array}$ & $\begin{array}{l}1284,9 \\
(342,4)\end{array}$ & ns \\
\hline $\begin{array}{l}\text { Produção total de leite ajustada para } 270 \mathrm{~d} \\
\text { (kg/animal/lactação)* }\end{array}$ & $\begin{array}{l}1040,4 \\
(190,2)\end{array}$ & $\begin{array}{l}1144,5 \\
(186,9)\end{array}$ & $\mathrm{ns}$ \\
\hline
\end{tabular}

Portanto, conclui-se que a manipulação diária dos animais empregada neste experimento afeta alguns parâmetros reprodutivos, comprometendo o número de coberturas por concepção, mas não interfere no intervalo partoconcepção, na taxa de prenhez ao final da estação e na produção leiteira, considerando-se o sistema de manejo e índices de produção do experimento.

\section{AGRADECIMENTOS}

Os autores agradecem à Empresa Brasileira de Pesquisa Agropecuária - Embrapa, à União Pioneira de Integração Social - UPIS Faculdades Integradas e à Universidade de Brasília - UnB pelo suporte financeiro. 


\section{COMITÊ DE ÉTICA}

Este trabalho foi aprovado pela Comissão de Ética no Uso de Animais da UPIS-Faculdades Integradas (CEUA-UPIS), protocolo 062/11.

\section{REFERÊNCIAS}

ABDALLA, E.B. Improving the reproductive performance of Egyptian buffalo cows by changing the management system. Animal Reproduction Science, v. 75 , p. 1-8, 2003.

ANDRIGHETTO, C.; JORGE, A.M.; GOMES, M.I.F.V., HOCH, A.; PICCININ, A. Efeito da Monensina Sódica sobre a Produção e Composição do Leite, a Produção de Mozzarela e o Escore de Condição Corporal de Búfalas Murrah. Revista Brasileira de Zootecnia, v. 34, n. 2, p. 641-649, 2005.

BARUSELLI, P.S.; MUCCIOLO, R.G.; VIANA, W.G.; CASTRO JUNIOR, F.G.; REICHERT, R.H.; ALVAREZ, R.H. Involução uterina no período pós-parto em fêmeas bubalinas (Bubalus bubalis). Boletim de Indústria Animal, v. 53, p. 51-55, 1996.

BERNARDES, O. Bubalinocultura no Brasil: situação e importância econômica. Revista Brasileira de Reprodução Animal, v. 31, n. 3, p. 293-298, 2007.

CAMPANILE, G.; BARUSELLI, P. S.; VECCHIO, D.; PRANDI, A.; NEGLIA, A.; CARVALHO, N.A.T.; SALES, J.N.S.; GASPARRINI, B., D'OCCHIO, M.J. Growth, metabolic status and ovarian function in buffalo (Bubalus bubalis) heifers fed a low energy or high energy diet. Animal Reproduction Science, v. 122, p. 74-81, 2010 .

HONPARKHE, M.; GHUMAN, S. P. S.; DADARWAL, D.; SINGH, J.; DHALIWAL, G. S.; SINGH, N. Influence of rectal massage of ovaries in anestrus buffaloes. Indian Journal of Animal Sciences, v. 78, n. 12, p. 1376-1377, 2008.

JORGE, A. M., GOMES, M. I. F. V.; HALT, R. C. Efeito da utilização da somatotropina bovina recombinante (bST) sobre a produção de leite em búfalas. RevistaBrasileira de Zootecnia, v. 31, n. 3, p. 1230-1234, 2002

MWAANGA, E. S.; ZDUŃCZYK, S.; JANOWSKI, T. Comparative study on the efficacy of hormonal and non hormonal treatment methods in ovarian afunction affected dairy cows. Bulletin of the Veterinary Institute in Pulawy, v. 48, p. 265-267, 2004. Disponível em: http://www.piwet.pulawy.pl/doc/biuletyn 483/16 mwaanga.pdf >. Acesso em: 22 jul. 2011.

PAUL, S. S; LAL, D. Nutrient requirements of buffaloes. Delhi: Satish Serial, 2010. p. 6-7. ISBN 8189304-76-3.

RASSI, L. F.; ARAUJO, V. C.; VASCONCELLOS, B. F.; NASCENTE, F. X.; SCHWABACHER, V. G.; MOREIRA, P. C. Correlação entre produções parciais e totais de leite em um rebanho bubalino. Estudos, v. 36. n. 11/12, p. 1135-1139, 2009.

SAMPAIO NETO, J. C.; MARTINS FILHO, R.; LÔBO, R. N. B.; TONHATI, H. Avaliação dos desempenhos produtivo e reprodutivo de um rebanho bubalino no Estado do Ceará. Revista Brasileira de Zootecnia, v. 30, n. 2, p. 368-373, 2001.

SILVA, M. M. A; BARROS, N. A. M. T.; RANGEL, A. H. N.; FONSECA, F. C. E.; VELOSO JR, F.; LIMA JR, D. M.Persistência da lactação em búfalas da raça Murrah (Bubalus bubalis) exploradas no agreste do Rio Grande do Norte. Acta Veterinaria Brasilica, v. 4, n. 4, p. 286-293, 2010.

STATSOFT, INC. STATISTICA (data analysis software system), version 7, 2004. www.statsoft.com.

USMANI, R. H.; DAILEY, R. A.; INSKEEP, E. K. Effects of limited suckling and varying prepartum nutrition on postpartum reproductive traits of milked buffaloes. Journal of Dairy Science, v. 73, n. 6, p. 15641570,1990 .

VALE, W. G.; RIBEIRO, H. F. L. Características reprodutivas dos bubalinos: puberdade, ciclo estral, involução uterina e atividade ovariana no pós-parto. Revista Brasileira de Reprodução Animal, v. 29, n. 2, p. 63-73, 2005. 